\title{
Application specific extension of the MCRI: Memory Colors and Preferred Colors of Reddish Meat Products
}

\author{
Kevin A.G. Smet ${ }^{* a}$, Inge Roelandts ${ }^{\text {, }}$, Kees Teunissen ${ }^{\text {, }}$, Simone Poort ${ }^{\text {b }}$, Peter Hanselaer ${ }^{\text {a }}$ \\ ${ }^{a}$ ESAT/Light\&Lighting Laboratory, KU Leuven, BE \\ ${ }^{b}$ Signify (formerly known as Philips Lighting), Eindhoven, $N L$ \\ * Corresponding author: Kevin.Smet@kuleuven.be
}

\begin{abstract}
This paper explores the possibility of extending the memory color rendition index (MCRI) for specific applications. The memory colors and preferred colors of three types of meat products (ham sausage, chicken breast and raw hamburger) have been derived in a psychophysical rating experiment. The rating data have been modelled by modified bivariate Gaussian functions from which similarity functions have been derived. These functions, which predict the degree of similarity with respect to the memory color or preferred color of the meat products, have been used to evaluate the color quality of four commercially available meat lighting lamps. It was found that predicted color quality depends on the illuminance level, indicating that illuminance level is a potentially important aspect of color rendition evaluation, one that is not taken into account by most color rendition indices.
\end{abstract}

Keywords: memory colors, preferred colors, color quality, color rendition, meat lighting, MCRI

\section{Introduction}

The concept of memory color refers to the color associated with a familiar object in (long term) memory ${ }^{1}$. It should be distinguished from color memory, which is the ability to recollect colors in general. Memory colors were first introduced in the late $19^{\mathrm{e}}$ century by Hering when he stated that we view the world through the spectacles of memory ${ }^{2}$. Since then, memory colors and preferred colors - the color one would like objects to have - have long been of interest to many different areas of color research ${ }^{1,3-7}$. They have been suggested or used as an internal reference to assess object color appearance and color quality in color reproduction and color rendering. Judd's flattery index ${ }^{4}$ and Thornton's color preference index ${ }^{5}$ developed in the sixties and seventies were based on memory data obtained by Sanders ${ }^{3}$ and Newhall et al. ${ }^{8}$. More recently, the use of memory colors as an internal reference has been regained attention by the publication of a memory color based color rendering index (MCRI) ${ }^{7,9}$. Very recently, memory colors have been used in chromatic adaptation research to determine corresponding colors and to model the degree of adaptation ${ }^{6,10}$. This paper reports on a study ${ }^{11,12}$, performed for Signify (formerly known as Philips Lighting), where the memory and preferred colors have been determined for three types of meat with different degrees of 'redness': ham sausage, chicken breast and raw hamburger. To illustrate the potential of extending the Memory Color Rendition Index (MCRI) with application specific color rendition indices, the psychophysical data was used to derive three similarity functions, one for each meat product. Similarity functions are the key components of the MCRI; they describe how similar any object color appears to the memory (or preferred) color. Finally, these three similarity functions were then used to evaluate the quality of four commercially available light sources with respect to the illumination of meat.

\section{Methodology}

\subsection{Experimental setup}

The setup was similar to the original one used to develop the Memory Color Rendition Index ${ }^{9,13}$. The setup consisted of a specially designed, calibrated, illumination box that allowed to change the 
apparent color of the meat product by illuminating it with colored light emitted by three Red-GreenBlue-Amber LED modules (see Fig. 1). The meat was placed between two parallel transparent glass plates in front of a $3500 \mathrm{~K}$ self-luminous back panel $\left(L=47.2 \mathrm{~cd} / \mathrm{m}^{2}, x=0.4134, y=0.4221\right)$ that provided a steady adaptation state. The glass plate on top served to prevent dehydration and subsequent discoloration. To mask cues to the color of the illumination, the glass plates were oriented such that the mirrored (specular) reflection of LED sources could not be seen. The illumination box was closed, with the exception of a small viewing aperture, surrounded at the exterior side by a white front panel. The front panel was illuminated by an external light source and had approximately the same chromaticity $\left(L=198.1 \mathrm{~cd} / \mathrm{m}^{2}, x=0.3863, y=0.3817\right)$ as the back panel.
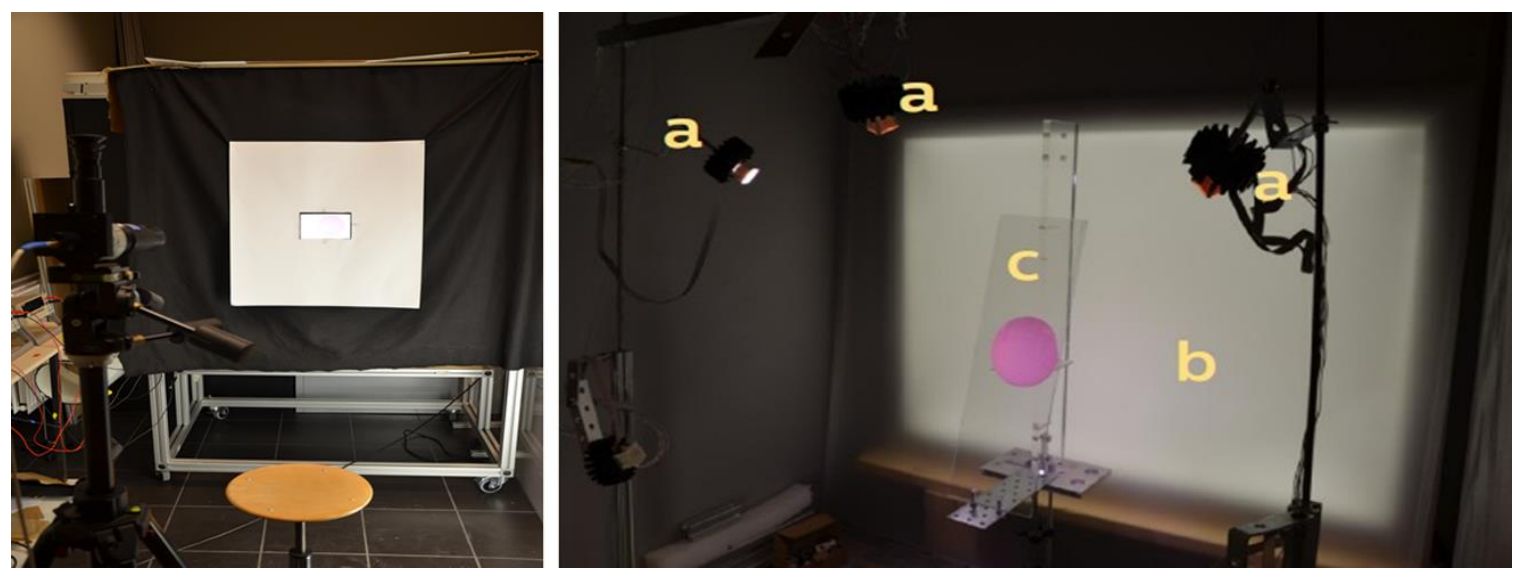

Fig. 1. Experimental setup. Left: As viewed by an observer. Right: Inside of illumination box. a: RGBY leds to change meat color; b: $3500 \mathrm{~K}$ self-luminous back panel to provide steady adaptation state; $c$ glass plates to hold meat and to protect it from dehydration and discoloration.

\subsection{Meat products}

The following three types of meat were selected: ham sausage, chicken breast and raw hamburger (see Fig. 2 left). The choice of meat types was based on spatial uniformity, familiarity of the test subjects with the meat and a sufficient variation in chromaticity between the different types of meat. The spectral reflectance and the chromaticity under illuminant D65 and d:8 geometry are shown in resp. the middle and right-hand graphs of Fig. 2.
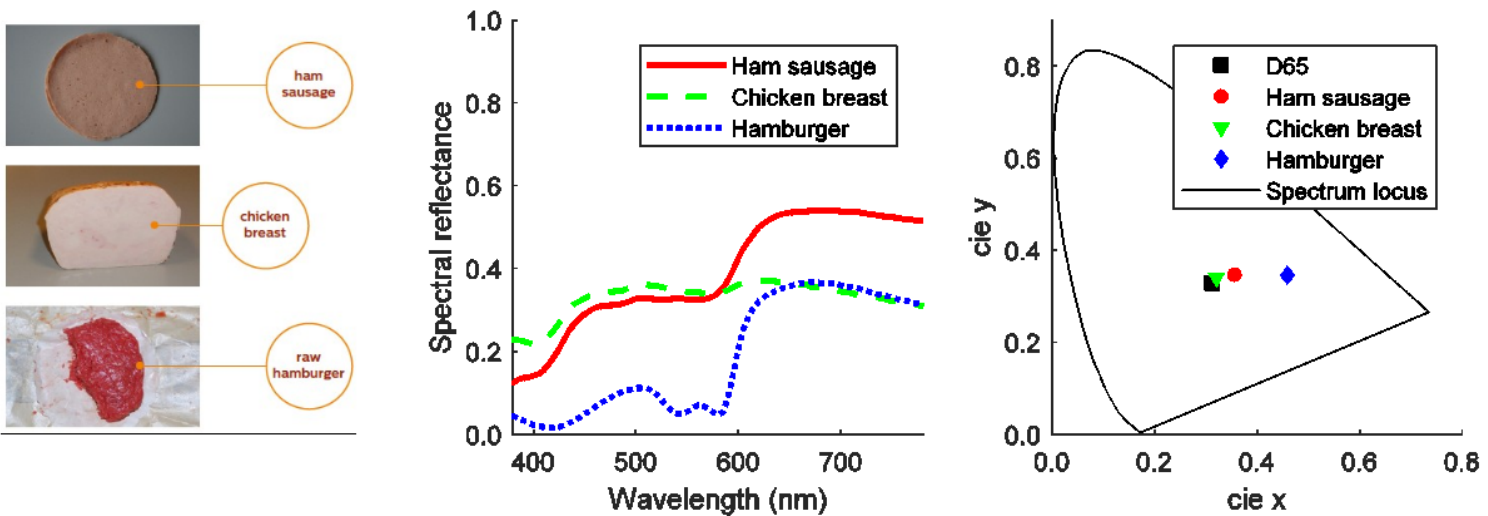

Fig. 2. Left: Photos of the three meat products. Middle: Spectral reflectance (red solid line: ham sausage, green dashed line: chicken breast, blue dotted line: raw hamburger). Right: CIE x,y chromaticity coordinates (black square: D65, red circle: ham sausage, green triangle: chicken breast, blue diamond: raw hamburger, black solid line: spectrum locus).

\subsection{Experimental procedure}

Each meat product was presented to an observer in more than 100 different colors, but at approximately the same luminance (ham sausage: $L_{10}=354 \pm 14 \mathrm{~cd} / \mathrm{m}^{2}$; chicken breast: $L_{10}=433 \pm 16 \mathrm{~cd} / \mathrm{m}^{2}$ and raw hamburger: $\left.L_{10}=136 \pm 15 \mathrm{~cd} / \mathrm{m}^{2}\right)$. The observer was asked to rate 
each presented color on a $0-10$ scale in terms of what they remember it to look like (memory) and in terms of attractiveness.

The colors presented for each type of meat were selected at random from a grid of predefined, uniformly distributed chromaticity points. The number of points in the grids for ham sausage, chicken breast and raw hamburger was resp. 114, 127 and 123. To determine the observed chromaticity of the presented color as accurately as possible, the spectral radiance of the stimulus was measured after each visual assessment using an OceanOptics QE65000 spectrometer coupled to Bentham radiometric measuring head. Luminance and chromaticity values were determined using the CIE $196410^{\circ}$ observer as the objects had a field of view larger than $4^{\circ}$. Note that each observer was presented with the same randomized sequence to minimize deviations in object chromaticity (standard deviation of $\Delta E u^{\prime}{ }_{10} v^{\prime}{ }_{10}=0.0014$ ) due to LED heating effects, as a preliminary study had shown that different random sequences lead to substantially different chromaticity values. Effects of meat discoloration during an experiment were found to be negligible for the ham sausage and chicken breast $\left(\Delta E u^{\prime}{ }_{10}, v^{\prime}{ }_{10}<0.003\right)$, but not for the raw hamburger $\left(\triangle E u^{\prime}{ }_{10}, v^{\prime}{ }_{10} \approx 0.008\right)$. The data for the raw hamburger were processed in a different way as for the ham sausage and chicken breast.

\subsection{Observers}

Each meat type was evaluated by a panel of 15 to 20 observers with an average age of 34 years and an average male-to-female ratio of 1.8. All observers had normal color vision as tested with the 24plate Ishihara test.

\subsection{Average observer derivation}

In case of the ham sausage and chicken breast, the chromaticity coordinates of the sampling grid were sufficiently stable (negligible effect of discoloration) and an average observer was constructed by averaging the rating data of each grid point over all observers. However, for the raw hamburger, discoloration was non-negligible: the average size of the covariance ellipses of the CIE $1976 u$ ' $v$ ' measured $10^{\circ}$ chromaticity coordinates for all observers was 0.0102 , which is considerably larger than a JND (just-noticeable-difference $\approx 0.003^{14}$ ). An average observer was therefore created as follows. First a uniform mesh of circular 'pixels', with a radius of $0.003 u$ 'v' units, was generated in the CIE $1976 u v^{\prime} v^{\prime} 10^{\circ}$ chromaticity diagram. For each observer, the average chromaticity and observer rating within each pixel was calculated. Finally, a rating set for the average observer was determined by averaging each pixel's data over all observers using the arithmetic mean.

\section{Results and discussion}

\subsection{Observer variability}

General inter and intra observer variability was evaluated using the Standardized-Residual-Sumof-Squares (STRESS) ${ }^{15}$ and the average Root-Mean-Square-Error (RMSE). Inter-observer variability was calculated by comparing each observer's ratings with that of the average observer and averaging over all observers. Intra observer variability was estimated from three repeat experiments by three observers for each meat type. For each observer, the variability between each repeat experiment's ratings and the average ratings was determined. Those variability values were then averaged over all the repeat experiments and the three observers to obtain a general measure for the intra observer variability.

No intra observer variability was calculated for the raw hamburger as discoloration prevented showing the same chromaticity points.

The STRESS and RMSE inter- and intra-observer variability for the different meat types is given in Table 1. Note that the intra observer variability for the raw hamburger was not calculated because of considerable changes in the chromaticity coordinates due to discoloration. 
Table 1. Inter- and intra-observer variability. M: memory color, P: preferred color.

\begin{tabular}{|c|c|c|c|c|c|c|c|c|}
\hline & \multicolumn{4}{|c|}{ Intra } & \multicolumn{4}{|c|}{ Inter } \\
\hline & \multicolumn{2}{|c|}{ STRESS } & \multicolumn{2}{|c|}{$R M S E$} & \multicolumn{2}{|c|}{ STRESS } & \multicolumn{2}{|c|}{ RMSE } \\
\hline & M & $\mathbf{P}$ & $\mathbf{M}$ & $\mathbf{P}$ & $\mathbf{M}$ & $\mathbf{P}$ & $\mathbf{M}$ & $\mathbf{P}$ \\
\hline Ham sausage & 0.31 & 0.27 & 1.1 & 1.0 & 0.43 & 0.39 & 1.9 & 1.7 \\
\hline Chicken breast & 0.18 & 0.20 & 0.8 & 0.8 & 0.41 & 0.44 & 2.0 & 2.1 \\
\hline Raw hamburger & - & - & - & - & 0.40 & 0.37 & 2.0 & 1.9 \\
\hline
\end{tabular}

From Table 1, it is clear that the inter-observer STRESS and RMSE values are very similar for all meat types. The intra- and inter-observer STRESS values are also similar to the values reported in a study on cross-cultural variation in memory colors ${ }^{16}$ and indicate good observer consistency and agreement. The intra observer $R M S E$ values show that observers are capable of reporting the same rating within about one scale unit. The agreement between observers was approximately two units.

\subsection{Memory colors, preferred colors and similarity functions}

For each meat type $i$, similarity functions, $S_{\mathrm{i}}\left(x_{10}, y_{10}\right){ }^{13}$ were derived by pooling the rating data (as described in previous section) and modeling it with a modified bivariate Gaussian rating function, $R_{i}\left(x_{10}, y_{10}\right)$ (see Eq. 1):

$$
\begin{aligned}
& d\left(x_{10}, y_{10}\right)^{2}=\left(\begin{array}{l}
x_{10}-a_{3} \\
y_{10}-a_{4}
\end{array}\right)^{T}\left(\begin{array}{ll}
a_{1} & a_{5} \\
a_{5} & a_{2}
\end{array}\right)\left(\begin{array}{l}
x_{10}-a_{3} \\
y_{10}-a_{4}
\end{array}\right) \\
& S\left(x_{10}, y_{10}\right)=e^{-0.5 \cdot d\left(x_{10}, y_{10}\right)^{2}} \\
& R\left(x_{10}, y_{10}\right)=a_{7}+a_{6} \cdot S\left(x_{10}, y_{10}\right)
\end{aligned}
$$

With $a_{1-7}$ and $x_{10}, y_{10}$ representing the model parameters and chromaticity coordinates respectively. The model parameters $a_{3-4}$ correspond to the centroid of the bivariate Gaussian function and represent the memory or preferred color; $a_{1,2,5}$ determine the shape, size and orientation of the bivariate Gaussian function and $a_{6}$ and $a_{7}$ are linear scaling parameters to convert the $0-1$ range of the similarity function $S_{\mathrm{i}}\left(x_{10}, y_{10}\right)$ to the rating scale of the average observer. In this study, data was modelled in the CIE $1964 x_{10}, y_{10}$ chromaticity diagram because other, more uniform chromaticity systems (such as $u^{\prime} v^{\prime}$ or IPT ${ }^{17}$ ) did not influence the goodness-of-fit considerably $\left(R^{2}\right.$ and $R M S E$ differences with that of CIE $x y$ were less than 0.006 and 0.02 , respectively). While the rating functions can be used to predict the rating an observer would give to an arbitrary chromaticity point when asked to rate one of the meat products, the similarity functions provide a convenient measure of the similarity of the arbitrary chromaticity point to their memory and preferred colors. This similarity can then be used to evaluate the color quality of a light source spectrum, whereby it is assumed that higher similarity corresponds to higher lighting color quality. The three derived similarity functions therefore provide an application specific extension to the memory color rendition index (MCRI).

The similarity functions for memory color and preferred color for each of the three meat types are plotted in Fig. 3. The goodness-of-fit has also been analyzed by calculating the STRESS, RMSE and $R^{2}$ values between model predictions and observer ratings. These results are shown in Table 2 .

Table 2. Goodness-of-fit between rating model predictions and observer ratings in terms of STRESS, $\underline{R M S E}$ and $R^{2}$. M: memory color, P: preferred color.

\begin{tabular}{ccccccc}
\hline & \multicolumn{2}{c}{ STRESS } & \multicolumn{2}{c}{$\boldsymbol{R M S E}$} & \multicolumn{2}{c}{$\boldsymbol{R}^{\mathbf{2}}$} \\
& $\mathbf{M}$ & $\mathbf{P}$ & $\mathbf{M}$ & $\mathbf{P}$ & $\mathbf{M}$ & $\mathbf{P}$ \\
\cline { 2 - 7 } Ham sausage & 0.14 & 0.12 & 0.49 & 0.45 & 0.94 & 0.93 \\
Chicken breast & 0.12 & 0.14 & 0.48 & 0.52 & 0.90 & 0.88 \\
Raw hamburger & 0.29 & 0.27 & 1.20 & 1.11 & 0.86 & 0.77 \\
\hline
\end{tabular}



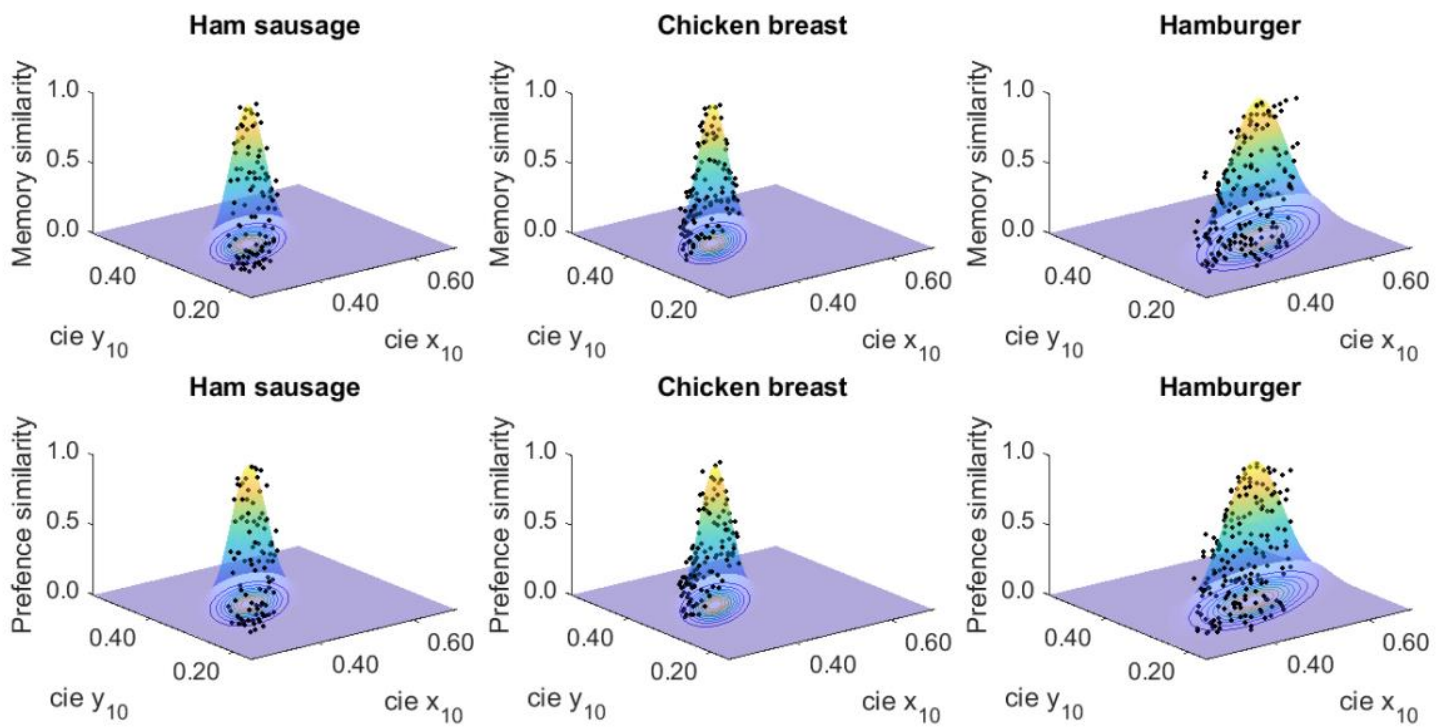

Fig. 3: Similarity functions. Top row: memory colors, Bottom row: preferred colors. From left to right: ham sausage, chicken breast, hamburger. Black dots: averaged observer scores rescaled to a 0-1 range.

From Fig. 3 and the goodness-of-fit measures in Table 2, it is clear that the rating models fit the data very well. The STRESS values are low, indicating good agreement. In addition, they are lower than the inter-observer variability, indicating the models are about as good as they get. This is also clear from the RMSE values, which for the ham sausage and the chicken breast are about half the unit of the $0-10$ scale adopted during the experiments. For the raw hamburger they are a little bit higher. The good fit of the models is confirmed by the high coefficients of determination $R^{2}$.

\subsection{Memory colors versus preferred colors}

For easy comparison, the cross-sections of the memory color and preferred color similarity bivariate Gaussian functions, at a similarity level of 50\%, have been plotted in Fig. 4.

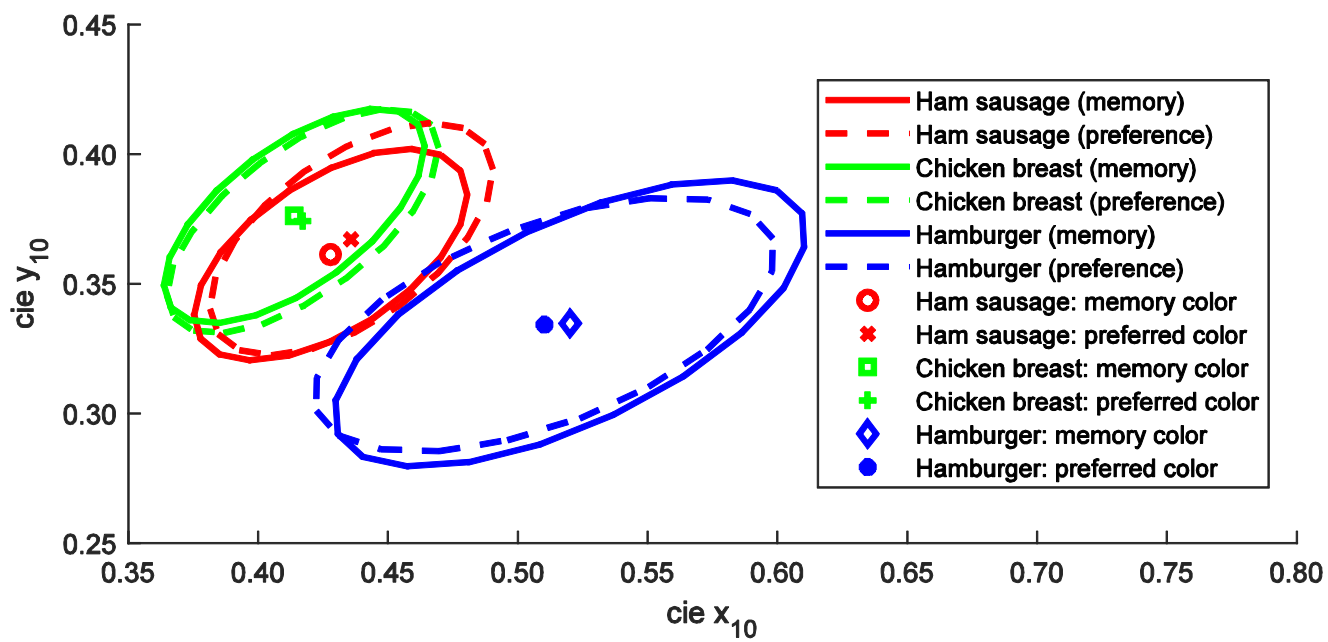

Fig. 4: Similarity function cross-sections at 50\% similarity. Solid lines: memory color, dashed lines: preferred color. Red, green and blue designate respectively: ham sausage, chicken breast and raw hamburger. Different markers have been used for the center of each similarity function: red circle / x: memory/preferred color of ham sausage; green square / +: memory/preferred color of chicken breast; blue diamond/dot: memory/preferred color of raw hamburger.

The color difference $\left(\Delta u^{\prime}{ }_{10} v^{\prime}{ }_{10}\right)$ between the two centers of the similarity functions for memory color and preferred color are $0.0029,0.0046$ and 0.0046 for the ham sausage, chicken breast and raw hamburger respectively. For the latter two, this is greater than a just-noticeable difference 
$\left(\Delta u^{\prime}{ }_{10} v^{\prime}{ }_{10} \approx 0.003\right)$, but is still within a 7-step MacAdam ellipse. For the meat products in this study, memory colors seem to be a very good predictor for preferred colors. This is also evident from the high $R^{2}$ values between the memory color and preferred color ratings, which are $0.94,0.92$ and 0.93 for the ham sausage, chicken breast and raw hamburger respectively.

\section{Application: evaluation of color quality of four commercially available meat lighting lamps.}

The color quality of four commercially available meat lighting lamps was mathematically evaluated in terms of similarity with meat product memory color and preferred color.

\subsection{Lamp details}

Two lamps, Red Premium and Rosé, are meat lighting specialty products from Signify (formerly known as Philips Lighting). For comparison, two common lamp spectra, also used in meat lighting, were included in the color quality evaluation. The spectra and some colorimetric characteristics of the four lamps are given in respectively Fig. 5 and Table 3.

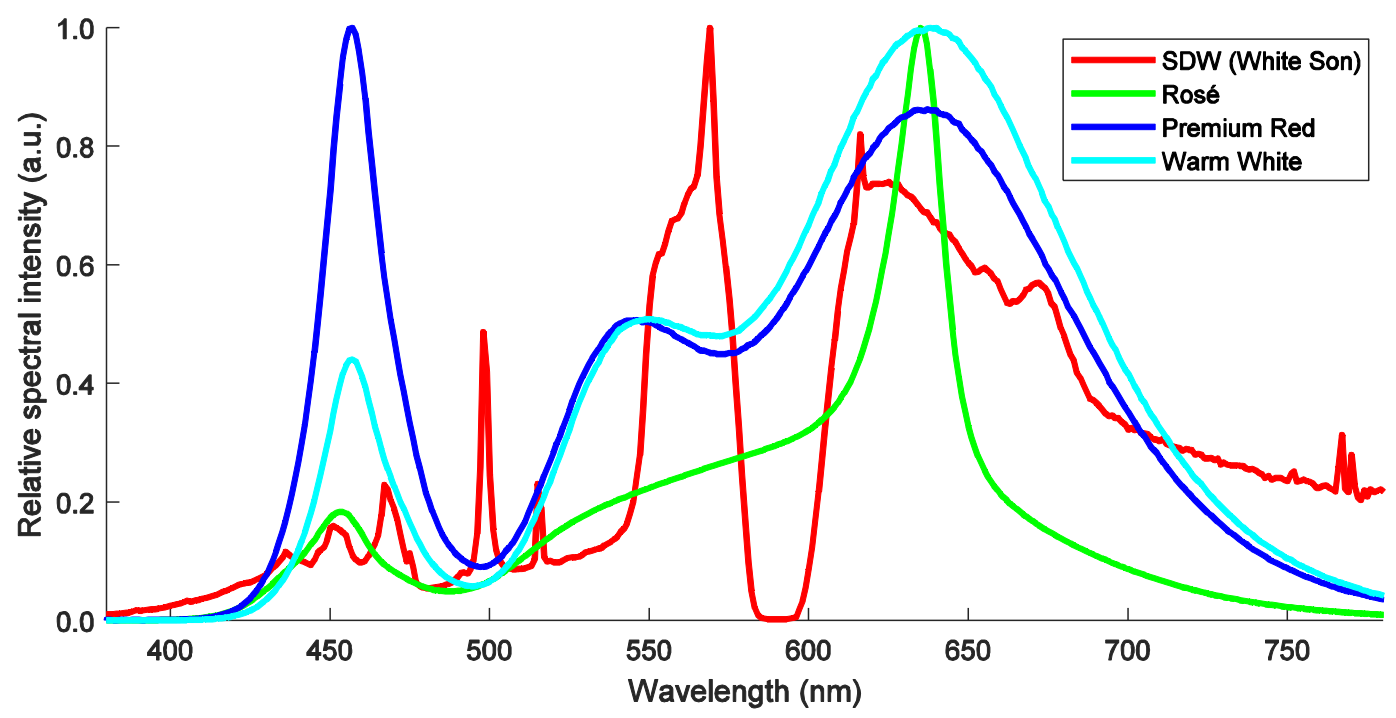

Fig. 5. Spectral power distribution of the four commercially available meat lighting lamps.

Table 3. Colorimetric details of the four meat lighting lamps. CCT: correlated color temperature; CIE 13.3-1995 $R_{\mathbf{a}}{ }^{18}$ and MCRI $R_{m}(\text { at } 500 \mathrm{~lx})^{9}$.

\begin{tabular}{cccc}
\hline & CCT (K) & CIE $\boldsymbol{R}_{\boldsymbol{a}}$ & MCRI $\boldsymbol{R}_{\boldsymbol{m}}$ \\
\hline SDW (White SON) & 2717 & 81 & 86 \\
Rose & 2215 & 92 & 85 \\
Premium Red & 3110 & 89 & 94 \\
Warm White & 2420 & 95 & 87 \\
\hline
\end{tabular}

\subsection{Color quality evaluation: material and methods}

The assumption is that increased similarity with memory or preferred colors results in higher color quality, which has resulted, in the case of the MCRI, in color quality predictions that are highly correlated with visual evaluations ${ }^{7,19}$.

Similar to the MCRI, specific color quality indicators, $S i$, one for each specific meat type $i$, can be obtained as the function values of the three similarity functions $\operatorname{Si}(\mathrm{x}, \mathrm{y})$ with the $x, y$ chromaticity coordinates of the meat product under the test light source taken as input ${ }^{9}$. Chromaticity coordinates are calculated using the CIE $196410^{\circ}$ color matching functions, the object spectral reflectance (see Fig. 2) and the light source spectral power distributions (see Fig. 5). A general color quality indicator, $S_{a}$, is defined as the geometric mean of the specific indicator values of each meat product. More details on the calculation of color quality indicators, $S_{i}$ and $S_{a}$ can be found in ${ }^{9}$. 
To account for different viewing conditions, prior to calculating the specific indicator values, the meat chromaticity coordinates under the test light source, are transformed to the corresponding chromaticity coordinates under the experimental conditions using the CAT02 chromatic adaptation transform ${ }^{20}$. A two-step transform ${ }^{10}$ is adopted: a first transform from the test source to a baseline illuminant condition (the equi-energy illuminant), followed by a final transform from the latter to the experiment condition. Each step is characterized by its own specific degree of adaptation, which are calculated from the two sets of viewing conditions. The test conditions are assumed to have as white point the chromaticity of the test source, an average surround $(F=1)$ and a background luminance factor $Y_{b}$ of 20. The latter is used to calculate the adapting field luminance from the illuminance. Just as in the MCRI, color quality is therefore also dependent on the test source illuminance, which in this study has been varied from 250 lux to $1000 \mathrm{~lx}$ in steps of $250 \mathrm{~lx}$. The experimental conditions were characterized by a dim surround $(F=0.9)$, a background luminance factor of 90 (white paper of front panel) and a white point of $3500 \mathrm{~K}\left(X_{10} Y_{10} Z_{10}=217.6,209.8\right.$, 128.3).

\subsection{Color quality evaluation: results}

For all four light sources, the degrees of similarity with the memory color and preferred colors, i.e. the specific color quality indicator values, have been plotted as a function of illuminance in Fig. 6 . The top and bottom rows are the results for degree of similarity with respectively the meat type's memory color and preferred color, with meat type varying column-wise. It is clear that all four light sources have a very high similarity score ranging between approximately 0.80 and 1.00 and that the degree of similarity is dependent on the illuminance level due to changes in the chromatic adaptation state of an observer. For nearly all subplots, the degree of similarity rises slightly with the illuminance level for nearly all light sources. The general color quality indicators (calculated as the geometric mean of the specific indicators for each meat type) of the four light sources are plotted in Fig. 7 together with the memory color rendition general indicator (MCRI Sa) values ${ }^{9}$. It is clear that the meat lighting lamps score higher in terms of the general color quality indicator specifically obtained for meat products than in terms of the MCRI Sa, indicating that the lamps serve their purpose, i.e. illumination of meat. It can also be seen that the meat $\mathrm{Sa}$ values show a similar relative trend as a function of illuminance level to the MCRI Sa values. The relative magnitude of the Sa values for the different lamps is however different. While the Red Premium is the clear best with regards to MCRI (see also Table 3), the warm-white phosphor LED has a very similar (but still slightly lower) performance when the lamps are evaluated specifically for meat products. It can also be seen that in some cases the order of lamp performance changes at a particular illuminance level. For example, at around 500 lux, the white SON lamp is overtaken by the Rosé lamp in terms of MCRI Sa and the warm-white phosphor LED becomes slightly higher performance than the Red Premium lamp in terms of preferred color (but not memory color). Similar swaps in quality, although much less pronounced visually due to the high Sa values, also occur for the meat Sa, but at higher illuminance levels (near 1000 lux). It is clear that illuminance can play an important role in color quality evaluation, an aspect which is not generally taken into account by most color rendition indices (except MCRI). 

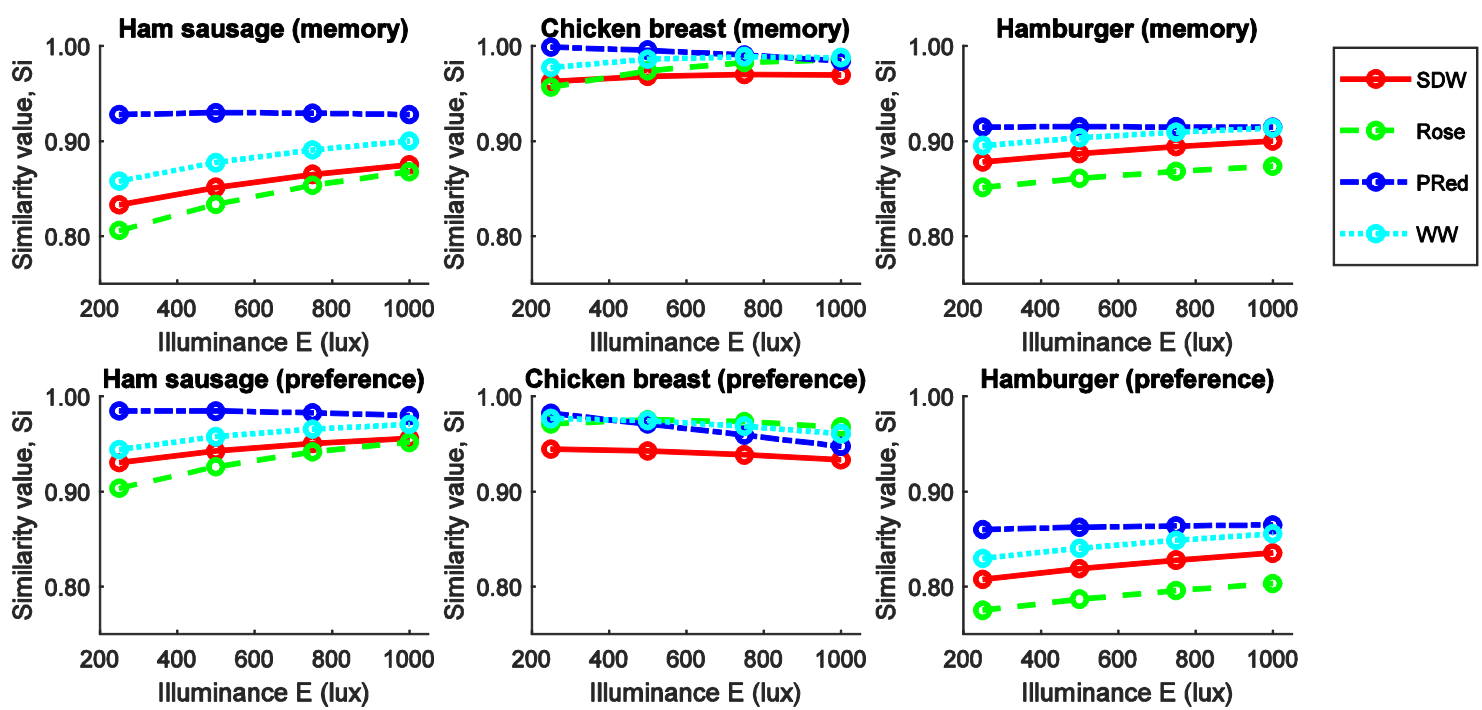

Fig. 6: Lamp performance in terms of similarity with memory color and preferred color. Top row: memory colors, Bottom row: preferred colors. From left to right: ham sausage, chicken breast, hamburger. Solid red line: SDW (White SON), dashed green line: Rosé, blue dash-dotted line: Premium Red and cyan dotted line; warm phosphor white LED.
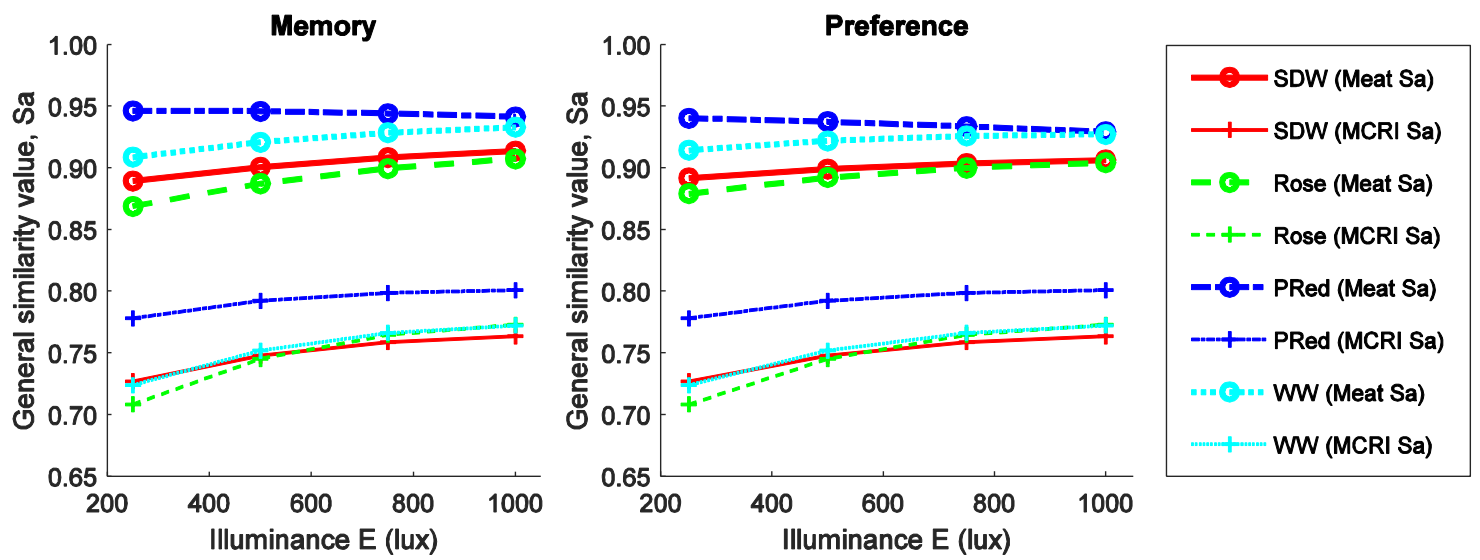

Fig. 7: General lamp color quality. Left: memory colors, Right: preferred colors. Solid red line: SDW (White SON), dashed green line: Rosé, blue dash-dotted line: Premium Red and cyan dotted line; warm phosphor white LED. Lines with circles denote Sa for meat, lines with + denote MCRI Sa values.

\section{Conclusions}

The memory colors and preferred (attractiveness) colors for three types of meat products with various degrees of redness were investigated. The obtained rating data can be fitted well with a modified bivariate Gaussian model. It is found that the memory color is closely related to the preferred color and the concept of memory color seems to be applicable to specialty applications whereby a good color appearance of specific products is crucial. In general, memory and preferred colors are slightly more saturated than the chromaticity observed as "natural" ${ }^{13}$.

Based on the rating data similarity functions were derived that predict the degree of similarity with respect to the memory color or preferred color of the meat products. These functions were used to evaluate the color quality of four commercially available meat lighting lamps. It was found that predicted color quality depends on the illuminance level, indicating that illuminance level is a potentially important aspect of color rendition evaluation, one that is not taken into account by most color rendition indices. 


\section{References}

1. Bartleson CJ. Memory colors of familiar objects. J Opt Soc Am. 1960;50:73-77.

2. Hering. Grundzüge Der Lehre Vom Lichtsinn. Berlin: Springer-Verlag; 1920.

3. Sanders CL. Colour preferences for natural objects. J Illum Eng Soc. 1959;54:452-456.

4. Judd DB. A flattery index for artificial illuminants. J Illum Eng Soc. 1967;62:593-598.

5. Thornton WA. A Validation of the Color-Preference Index. J Illum Eng Soc. 1974;4(1):48-52. doi:10.1080/00994480.1974.10732288

6. Smet KAG, Zhai Q, Luo MR, Hanselaer P. Study of chromatic adaptation using memory color matches, Part I: neutral illuminants. Opt Express. 2017;25(7):7732-7748. doi:10.1364/OE.25.007732

7. Smet KAG, Hanselaer P. Memory and preferred colours and the colour rendition of white light sources. Light Res Technol. 2016;48(4):393-411. doi:10.1177/1477153514568584

8. Newhall SM, Burnham RW, Clark JR. Comparison of successive with simultaneous color matching. J Opt Soc Am. 1957;47(1):43-54. http://www.opticsinfobase.org/abstract.cfm?URI=josa-47-1-43.

9. Smet KAG, Ryckaert WR, Pointer MR, Deconinck G, Hanselaer P. A memory colour quality metric for white light sources. Energy Build. 2012;49(C):216-225. doi:10.1016/j.enbuild.2012.02.008

10. Smet KA, Zhai Q, Luo MR, Hanselaer P. Study of chromatic adaptation using memory color matches, Part II: colored illuminants. Opt Express. 2017;25(7):8350-8365. doi:10.1364/OE.25.008350

11. Roelandts I, Smet K, Hanselaer P. Whitepaper: Visual Test on Meat Perception. Ghent; 2014.

12. Poort S, Smet KAG, Bigot E. Lighting recipes for fresh food retailers. Webinar, Philips Lighting. http://www.lighting.philips.com/main/education/lighting-university/lightinguniversity-browser/webinar/fresh-food-lighting-recipes. Published 2016.

13. Smet K, Ryckaert WR, Pointer MR, Deconinck G, Hanselaer P. Colour appearance rating of familiar real objects. Color Res Appl. 2011;36(3):192-200.

14. Luo MR, Cui G, Georgoula M. Colour difference evaluation for white light sources. Light Res Technol. 2015;47(3):360-369. doi:10.1177/1477153514539696

15. Melgosa M, García PA, Gómez-Robledo L, et al. Notes on the application of the standardized residual sum of squares index for the assessment of intra- and inter-observer variability in color-difference experiments. J Opt Soc Am A. 2011;28(5):949-953. doi:10.1364/JOSAA.28.000949

16. Smet KAG, Lin Y, Nagy B V, et al. Cross-cultural variation of memory colors of familiar objects. Opt Express. 2014;22(26):32308-32328. doi:10.1364/OE.22.032308

17. Ebner F, Fairchild MD. Development and testing of a color space (IPT) with improved hue uniformity. In: IS\&T 6th Color Imaging Conference. Scottsdale, Arizona, USA; 1998:8-13.

18. CIE13.3-1995. Method of Measuring and Specifying Colour Rendering Properties of Light Sources. Vol CIE13.3-19. Vienna, Austria: CIE; 1995.

19. Smet K, Ryckaert WR, Pointer MR, Deconinck G, Hanselaer P. Correlation between color quality metric predictions and visual appreciation of light sources. Opt Express. 2011;19(9):8151-8166.

20. CIE16x-2004. A Review of Chromatic Adaptation Transforms. Vol CIE160-200. Vienna: CIE; 2004. 


\section{Author Bios}

Kevin A.G. Smet, obtained his PhD in Engineering at KU Leuven in 2011. He is currently appointed as an Associate Research Professor at KU Leuven. Kevin is the team leader of the Appearance \& Perception group of the Light\&Lighting Laboratory and is an active member of CIE Division 1. His research interests include color rendition and color quality of white light sources, color appearance modeling, memory and preferred colors, perception of light and color in virtual reality and color science in general.

Inge Roelandts has a Master in Engineering Science and in Engineering Technology. During the time of the project she worked at KU Leuven as a researcher. Since then she has moved on and is currently working as a manager for FARYS.

Kees Teunissen: Kees Teunissen obtained his Ph.D. degree in the field of psychophysics, perceptually relevant display characterization, from the Technical University of Delft, the Netherlands. He is a senior research scientist at Signify, formerly known as Philips Lighting, a visiting professor at the Southeast University, Nanjing, China, and President of the Dutch National Committee of the CIE. His research interests include researching quality of light aspects in products and systems, in particular related to color vision and color science and for safeguarding and optimizing user experience in integral lighting solutions.

Simone Poort: Simone Poort, obtained her PhD in Solid State Chemistry (Luminescence) at the University of Utrecht (NL) in 1997. In the same year she joined Philips Lighting (now Signify) and has been working as researcher and project leader on different lighting related topics like luminescence, (lamp) materials behavior, light quality and lighting application. Her current research includes the effect of light on the quality of fresh food.

Peter Hanselaer, full professor at KU Leuven, received his $\mathrm{PhD}$ in Solid State Physics at University of Gent (B) in 1986. In 1997, he founded the Light\&Lighting Laboratory, specialized in indoor lighting, metrology, optical design and visual perception. He is the Editor of CIE Division 1 and chair of the Advisory Committee of the Faculty of Engineering Technology, KU Leuven. Peter is teaching the master courses photonics, optical fibres and lighting. 